\title{
MECO Operation Compared: One and Two Bunches on an Aluminum and Two Bunches on a Titanium Conversion Target
}

J. W. Glenn

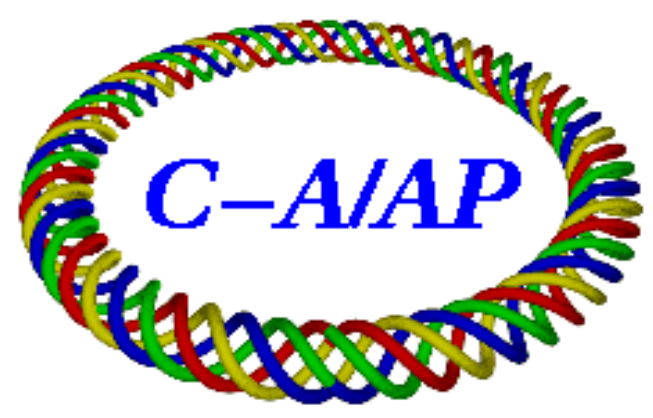

Collider-Accelerator Department Brookhaven National Laboratory Upton, NY 11973 


\section{MECO Operation Compared: \\ One and Two Bunches on an Aluminum and \\ Two Bunches on a Titanium Conversion Target}

J W Glenn

Introduction

MECO data rates are a function of AGS intensity, target material and the total time available while running for data taking. As the AGS intensity will be limited by the activation of the accelerators (ALARA Limits) the other parameters need optimization. The options examined are one or two bunches in the AGS extracted with an Aluminum conversion target and two bunches with a Titanium conversion target. Not only are these rates affected by the physics of capture and decay of the muons and data taking time, they are also strongly effected by the capabilities of the Booster, AGS, extraction and beam transport systems and their beam throughput limits due to activation. From rates provided by W Molson and limits by L Ahrens there appears to be an advantage in data rate by running the AGS with one bunch and using an Aluminum target for the same amount of accelerated protons.

There are other advantages to running with one bunch. While this note does not attempt to quantify the gains associated with these improvements, their qualities are described. There is expected to be a significant advantage for MECO due to the reduction of out of time (extinction) events by eliminating the main path for transfer of beam into adjacent buckets. Also there will not be time for the injected beam to blowup, thus making a brighter beam that will target better and reduce if not eliminate the need for larger aperture in the extraction and beam transport magnets. If this possibly is shown valid, we can reduce the cost to prepare the facility to run MECO by five to eight million dollars. The disadvantage to MECO of one bunch will be more live time and thus a higher cosmic ray background.

As an aside, I propose running another single bunch experiment to ramp up AGS intensities for a meaningful MECO engineering run. There is a low energy neutrino scattering experiment for which a letter of intent has been delivered and a proposal is being prepared that would be ideal for this mission. The possibility of running a higher intensity, lower energy beam out the fast extraction channel would need be shown reasonable for this experiment to be run as suggested. 
MECO Rate Comparison.

In order to better understand the problem, I modeled the possible rates. My results show that due to the expected improvement in muon capture, which is model dependant, two bunches on a Titanium target may give a better rate. This modeling is given in Appendix A.

The projections of MECO data rates for the three options of running were taken from a figure included in a note from W Molzen to P Pile ${ }^{1}$. I extracted numbers from these plots of relative rates for the three cases, normalized to two bunches on an Aluminum target with $40 \mathrm{TP} / \mathrm{Sec}$ throughput. The limits on AGS throughput were given me by L Ahrens ${ }^{2}$ as a spreadsheet that made assumptions on loss reduction resulting from various planned improvements to Booster and AGS. Combining the rates for a fixed amount of beam on the target for these cases predicted by W Molzon and the limits of AGS throughput by L Ahrens for these arrangements, the relative data rates can be predicted.

Table 1 Comparison of MECO Rates

\begin{tabular}{|r|r|r|r|r|}
\hline Setup & $\begin{array}{r}\text { Frac. Of } \\
\text { MECO } \\
\text { Goal }\end{array}$ & $\begin{array}{r}\text { Intensity } \\
\text { in AGS } \\
\text { for Goal } \\
\text { TP/P }\end{array}$ & $\begin{array}{r}\text { ALARA } \\
\text { Intensity } \\
\text { Limit } \\
\text { TP/Pulse }\end{array}$ & $\begin{array}{r}\text { Frac of } \\
\text { ALARA } \\
\text { for Goal }\end{array}$ \\
\hline Aluminum Target 2 Bunches in AGS & $100 \%$ & 40.0 & 42.4 & $94 \%$ \\
\hline Titanium Target 2 Bunches in AGS & $113 \%$ & 35.5 & 42.4 & $84 \%$ \\
\hline Aluminum Target 1 Bunch in AGS & $135 \%$ & 23.7 & 31.1 & $76 \%$ \\
\hline
\end{tabular}

\section{Comments.}

The numbers from Molzen were as given. A calculation is shown in Appendix A that may parallel his work and shows what assumptions could go into a comparisons. These results are similar to those given in Molzen's plots for Al targets, but they do show less beam is needed with a Titanium target.

The numbers from Ahrens are the result of a spread sheet that he built using the data presented in K Zeno's comparison of observations of Booster and AGS acceleration efficiencies over several years of high intensity running ${ }^{3}$. Zeno observed that his data, protons out verses protons in for both Booster and AGS fit well to a straight line with a positive offset. These offsets from the fit provide 'free 
protons', thus the limits for two Booster cycles per sec is higher than for one, but it is expected that efficiencies will drop with intensity in both accelerators. The allowable losses and the expected efficiencies are combined to give limits on beam delivered. With the completion of the Booster/AGS work for RSVP, the numbers of the allowable losses, and the expected efficiencies, are expected to increase. A statement in the attached comments to his evaluation mentioned that transfer efficiency would increase for a single bunch. I reduced the inefficiency by a factor of two and removed the positive offset for this effort. Caution is necessary in extrapolating beyond the measured intensities.

Advantageous side effects:

The rate of protons delivered to the MECO production target will be limited by the allowed loss rate in the Booster and AGS. Reducing the number of pulses per hour by almost a factor of two reduces the constriction of this limit, and allows more freedom to probe for more intensity per bunch above the best yet operationally achieved $12 \mathrm{TP} /$ Booster Pulse out of the AGS. This is a large benefit to MECO and is also of value to KOPIO.

AGS Injection would be at nearly full Bdot, as was done in a study of high brightness beams by Brennan, et. al. During the last high intensity run there were major losses in the H11 area. This was the vertical aperture for not understood reasons, but when this aperture was opened by moving the vertical orbit locally, equal vertical losses simply appeared elsewhere, and there was no increase in intensity latter in the cycle. Thus beam was lost on 70-75 $\mathrm{mm}$ apertures. If the beam had a truly gaussian tail, this would imply a beam emittance about twice what has been measured. Thus it appears that non-gaussian tails are being generating, and causing losses, on the 'flat bottom' (time required to achieve constant field in the AGS field, and then accept multiple loads from the Booster). It is expected that the transverse beam losses seen in the AGS would be greatly reduced as we see most of our vertical limiting losses on the flat bottom. We also see coherence shortly after injection, which would indeed increase effective emittance, thus we can assume this is where the transverse emittance blowup occurs. If the transverse emittance blowup can be reduced and the tails trimmed, the cost of increasing the vertical aperture of the extraction magnets and the switchyard can be 'scrubbed' saving the RSVP program some five to eight million dollars.

Most significantly for MECO, injecting on the fly creates a large band of unstable phase between full buckets and buckets required to be empty. Wall current 
monitor pictures taken by Ahrens show that beam leaked into adjacent buckets on the injection porch during the test run in '96. This unstable band would be about one half the phase width of the stable buckets. This would eliminate the opportunity of protons to transfer from the bucket that beam is injected into to adjacent, required to be empty, buckets at injection. As shown in the proposal, these 'baby bunches' in adjacent buckets were seen during targeting in the test run and would cause large background rates. If this can be fully demonstrated, the extinction measuring beam line could be reduced in scope 'scrubbing' more costs.

An advantage for the whole complex and particularly MECO is that fewer protons would be needed to run that program. This reduces the activation of the accelerator, extraction, transport, and target systems as well as the super conducting solenoid.

We could run the proposed low energy neutrino scattering experiment which needs moderately high intensity at MECO energies in the Fast Beam when the Booster and AGS are ready for higher intensity but before the slow beam lines and experiments are ready. High intensity single bunch operation could be fully developed and limits explored to provide a developed high intensity operation for MECO, as high intensity single bunch beam with small emittance would be required. We would be more ready for an RSVP engineering run, particularly for MECO, and would have demonstrated that the current Slow Beam apertures are adequate.

This run would not test the slow extraction system with the beam bunched in the RF buckets. We also need to develop bunched, low energy slow extraction as we have not ever run in this mode in the past, save for few short low intensity tests. The Booster and AGS injection work for RSVP high intensity need be done before regular high intensity running could commence, but if financed, some testing could be done sooner. If successful, the costs of aperture increases would be demonstrated to be unnecessary.

\section{Conclusion}

The operation of the AGS with only one bunch injected needs further tests. The payback for MECO includes higher data taking with lower peak detector rates and less extinction event problems. The payback for the AGS improvement part of RSVP is a multi-million dollar saving and simpler accelerator operation.

The following tests are suggested: 
The actual repetition rate for running with one and two bunches needs to be determined with this Main Magnet power supply. And then confirm with low intensity beam testing the stability of the injection field. The difference between the two rates may well be more than the assumed 0.2 seconds further increasing the advantages of running one bunch.

An accelerator physics study is needed to better understand beam loss, halo generation and emittance blowup with injection at constant AGS field and on rising field. This study while beneficial to RSVP is also of basic interest to the accelerator field. If beam core brightness can be increased, then RHIC luminosity for polarized protons would also increase. These tests can start with the bright, low emittance, ion beams available for RHIC operation. Later higher intensity protons would be needed for short tests. These tests would be at low energy as the blowup phenomena occur early in the AGS cycle minimizing accelerator activation.

Appendix A: A Model of Relative MECO Data Rates.

The assumptions for my model are first given with comment. Then calculation results are given. The data rate with a Titanium conversion target are about 50\% higher than rates gathered from data in Molzon's spreadsheet.

Table A1, Assumptions for Modeing Rates

\begin{tabular}{|c|c|c|c|c|c|c|c|c|c|c|c|c|}
\hline 1 & $\begin{array}{r}\text { Bnc } \\
\text { in } \\
A G S\end{array}$ & $\begin{array}{r}\text { AGS } \\
\text { Rep } \\
\text { Rate } \\
\text { Sec }\end{array}$ & $\begin{array}{r}\text { Ave } \\
\text { Stop } \\
\text { Time } \\
\\
\text { uSec }\end{array}$ & $\begin{array}{l}\text { Frac } \\
\text { Capt }\end{array}$ & $\begin{array}{r}\text { BR / } \\
\text { BR } \\
(A I)\end{array}$ & $\begin{array}{l}\text { Life } \\
\text { time }\end{array}$ & $\begin{array}{r}\text { Time } \\
\text { to } \\
\text { detec } \\
\text { on } \\
\text { uSec }\end{array}$ & $\begin{array}{r}9 \\
\text { Data } \\
\text { End } \\
\text { pre } \\
\text { next } \\
\text { bnch } \\
\text { uSec }\end{array}$ & $\begin{array}{r}10 \\
F M \\
\text { B Molz } \\
@ 20 T P \\
\text { const } \\
\text { TP } \\
\text { Bunch }\end{array}$ & \begin{tabular}{|r|}
11 \\
$F M$ \\
B Molz \\
@40TP \\
const \\
TP/Sec \\
\end{tabular} & $\begin{array}{r}\text { Max } \\
\text { AGS } \\
\text { TP/S } \\
\text { Leif } \\
\text { Ahrens } \\
\end{array}$ & \begin{tabular}{|r} 
Assum \\
Boost \\
to \\
AGS \\
eff \\
\end{tabular} \\
\hline Al & 2 & 1 & 0.37 & 0.6 & 1 & 0.88 & 0.65 & 0.04 & $100 \%$ & $100 \%$ & 40.4 & $85 \%$ \\
\hline $\mathrm{Ti}$ & 2 & 1 & 0.39 & 0.85 & 1.6 & 0.33 & 0.65 & 0.04 & $113 \%$ & $112 \%$ & 40.4 & $85 \%$ \\
\hline Al & 1 & 0.8 & 0.37 & 0.6 & 1 & 0.88 & 0.65 & 0.04 & $85 \%$ & $135 \%$ & 37.1 & $95^{\circ}$ \\
\hline
\end{tabular}

Columns $1 \& 2$ show the target and bunch number setup.

Column 3 is an assumed repetition rate of AGS cycle. The difference between 1 and 2 bunches in AGS may be greater.

Column 4 is from MECO Note $\# 44^{3}$ for Aluminum. The use of average time of stopping is inappropriate as the decay is exponential and the beam stops over 
a finite time. A slightly different effective stop time was calculated for

Titanium by comparing the decay integrals of actual distribution as shown in MECO \#44.

The fraction of muons captured (Column 5) was determined by a method given me by Bill Morse using the life times of free and captured muons.

The normalized conversion ratio (column 6) is as reported by Kitano, Koike \& Okada. $^{4}$

Life times (Column 7) are from note \#44.

Data start time (Column 8) is from the proposal ${ }^{5}$.

Data end time (Column 9), when data taking stops in anticipation of the next beam burst, is my estimate. A $50 \mathrm{nSec}$ bunch width and synchrotron frequency of $\sim 75 \mathrm{~Hz}$ results from an assumed extraction gamma of 8, rf Voltage of 200 $\mathrm{KV}$, and $5 \mathrm{eV}$-Sec emittance. The measured width (FW1\%M), as shown in the proposal, is $40 \mathrm{nSec}$; modeled width for the '97 PAC paper is $20 \mathrm{nSec}$. All this was at low intensity. In the proposal, there is no allowance in the live time for protons on the target, save the $20 \mathrm{nSec}$ flight time, before the center of the proton bunch. A $40 \mathrm{nSec}$ reduction in live time in addition to the $20 \mathrm{nSec}$ of flight time to the detector well may be required as the synchrotron frequency is only about $75 \mathrm{~Hz}$ so that voltage ripple on Flat Top would be non-adiabatic, causing longitudinal growth. This slightly improves the Aluminum target rates vs Titanium.

Results sent by W Molzon to P Pile are shown in columns $10 \& 11$, where 'FM' is figure of merit.

The Max TP/Sec due to ALARA in improved accelerators from spreadsheets used to generate Leif Ahrens's talk on 6 Jan 05 is given in Column 12.

Column 13 is my estimate of transfer efficiency between Booster and AGS for two bunches on a flat bottom of constant field and one bunch on the fly with a positive field ramp.

Resultant Calculated MECO Rates.

As the MECO detector is held off due to background for over half a microsecond after the proton beam hits the production target with two bunches in the AGS, MECO's duty factor of taking data during the spill is less than $50 \%$ of the half second in one spill time. Having only one bunch in the AGS increases the duty factor during the spill to nearly $75 \%$. Another factor on data rate is the AGS rep rate. Without a flat bottom of at least $0.2 \mathrm{Sec}$, it can run at least $20 \%$ faster. Combined, these duty factor increases improves overall live time by almost a factor of two. The smaller duty factor means that fewer than half the captured muons decay during live time. The Ti target captures more muons but almost half 
decay before the data gate opens, but then most of these are converted during live time. With an $\mathrm{Al}$ target with two bunches the gate on times are short enough that only half decay during the live time, but $\sim 15 \%$ of the muons survive to the gate opening time for the next bunch. With one bunch almost $90 \%$ of the muons decay during the live time, but the rates are low at the end of the gate time. These results are shown in Table A2.

Table A2: Calculated rates for MECO

\begin{tabular}{|c|c|c|c|c|c|c|c|c|c|c|c|c|}
\hline & $\begin{array}{r}\text { Bnc } \\
\text { in } \\
\text { AGS }\end{array}$ & $\begin{array}{r}\text { AGS } \\
\text { Rep } \\
\text { Rate } \\
\text { Sec }\end{array}$ & $\begin{array}{r}\text { Data } \\
\text { End } \\
\text { pre } \\
\text { next } \\
\text { bnch } \\
\text { uSec }\end{array}$ & $\begin{array}{c}\text { Bunch } \\
\text { period } \\
\text { uSec }\end{array}$ & $\begin{array}{c}\text { Live } \\
\text { time } \\
\text { bnch } \\
\text { uSec }\end{array}$ & $\begin{array}{r}\text { Frac } \\
\text { capt } \\
@ .65 \\
\text { uSec }\end{array}$ & $\begin{array}{l}\text { Frac } \\
\text { conv }\end{array}$ & \begin{tabular}{|} 
Rate I \\
$R($ Al 2) \\
const \\
TP in \\
AGS
\end{tabular} & $\begin{array}{r}\text { Rate } 1 \\
\text { R(Al 2) } \\
\text { const } \\
\text { TP/Sec }\end{array}$ & $\begin{array}{r}\text { AGS } \\
\text { Int } \\
\text { Late } \\
\text { for } \\
100 \% \\
\text { TP/P } \\
\end{array}$ & $\begin{array}{r}\text { Boost } \\
\text { Int } \\
\text { Late } \\
\text { for } \\
100 \% \\
T P / P \\
\end{array}$ & $\begin{array}{l}M \\
\text { Dn }\end{array}$ \\
\hline & 2 & 1 & 0.04 & 35 & 0.66 & & $9 \%$ & & & 40.0 & .5 & 16 \\
\hline 11 & 2 & 1 & & 35 & 0.66 & $63 \%$ & $55 \%$ & $186 \%$ & & & 8.3 & $0^{\circ}$ \\
\hline & 1 & 0.8 & 0.04 & .7 & 2.01 & $46 \%$ & $41 \%$ & $88 \%$ & $140 \%$ & 23.5 & 24.7 & $83^{\circ}$ \\
\hline
\end{tabular}

Cosmic Ray Back Ground and Initial Detector Rates

With one bunch the live time is increased, thus the Cosmic Ray background would increase, scaled from the proposal, from 0.0035 to 0.0069 events. If this is a problem, the low event rate times can be gated off during the spill, but more beam will be required per second on the target. Also shown below is the relative initial data rate. The initial detector data rate from the Titanium target increases by a factor of two for the same average data rate as for two bunches on an Aluminum target. The peak rate for one bunch is about the same as for two bunches on an Aluminum target. These results from the model are shown in Table 3A.

Table 3A. Initial Rates and Live Time.

\begin{tabular}{|r|r|r|r|r|r|r|}
\hline & $\begin{array}{r}\text { Bnc } \\
\text { in } \\
\text { AGS }\end{array}$ & $\begin{array}{r}\text { AGS } \\
\text { Rep } \\
\text { Rate }\end{array}$ & $\begin{array}{r}\text { Initial } \\
\text { Rate } \\
\text { Ri / } \\
\text { Ri of }\end{array}$ & $\begin{array}{r}\text { Duty } \\
\text { fact } \\
\text { on FT }\end{array}$ & $\begin{array}{r}\text { FT } \\
\text { duty } \\
\text { cycle }\end{array}$ & $\begin{array}{r}\text { Frac } \\
\text { Live } \\
\text { Time }\end{array}$ \\
\hline Al & 2 & 1 & $100 \%$ & $49 \%$ & $50 \%$ & $24 \%$ \\
\hline Ti & 2 & 1 & $236 \%$ & $49 \%$ & $50 \%$ & $24 \%$ \\
\hline Al & 1 & 0.8 & $97 \%$ & $74 \%$ & $63 \%$ & $47 \%$ \\
\hline
\end{tabular}


1 - Private communication W Molzon to P Pile on 12/27/'04.

2 - Private communication from L Ahrens, 2/3/'05

3 - 'Comparison of High Intensity Efficiencies- -' , K Zeno's, C-A/AP/\#86

4 - 'Choice of MECO Stopping Target And --', T.J. Lin, MECO-044

5 - 'Detailed calculation of lepton flavor violating muon-conversation rate for various nuclei', R. Kitano, M. Koike \& Y. Okada, Phys Rev D 66096002

6 - 'A Search for Mu N to e N --', W Molzon et.al., AGS Proposal P940 